\title{
Consumo de drogas en adolescentes: El papel del estrés, la impulsividad y los esquemas relacionados con la falta de límites
}

\section{Substance use in adolescents: The role of stress, impulsivity, and schemas related to lack of limits}

Esther Calvete; Ana Estévez
Departamento de Psicología. Universidad de Deusto

Enviar correspondencia a:

Departamento de Psicología. Universidad de Deusto, Apdo. 1,

48080, Bilbao. Tfno: 944139000

E-mail: ecalvete@fice.deusto.es

\section{RESUMEN}

Este estudió evaluó la asociación entre estrés, esquemas cognitivos, impulsividad y consumo de drogas en adolescentes. Una muestra de 657 adolescentes (367 chicas y 290 chicos) contestaron medidas de acontecimientos estresantes, esquemas cognitivos de Grandiosidad y Autocontrol Insuficiente, estilo impulsivo de resolución de problemas y consumo de drogas. Los resultados mostraron que los acontecimientos estresantes, y los esquemas de Grandiosidad y Autocontrol Insuficiente se asociaban significativamente al consumo. Además, el estilo impulsivo de resolución de problemas moderó la relación entre estresores y consumo, siendo esta mayor entre los más impulsivos. Por último, se encontraron diferencias en función del sexo en consumo y en esquemas cognitivos, obteniendo los chicos mayores puntuaciones que las chicas en Grandiosidad y en el consumo de marihuana, cocaína, LSD y éxtasis. Las chicas puntuaron más alto en consumo de tabaco. A pesar de estas diferencias, el sexo no moderó la asociación entre esquemas y el consumo.

Palabras clave: consumo de drogas, esquemas cognitivos, impulsividad, adolescentes, estrés.

\section{ABSTRACT}

The aim of this study was to assess the association between stress, cognitive schemas, impulsivity, and substance use in adolescents. A sample of 657 adolescents (367 girls and 290 boys) completed measures of stressful life events, cognitive schemas of grandiosity and insufficient self-control, impulsive style of problem-solving and substance use. The results indicated that stressful life events and the cognitive schemas were significantly associated with substance use. In addition, the impulsive style of problem-solving moderated the relationship between stressors and substance use, this association being stronger among more impulsive adolescents. Finally, the results indicated that boys scored higher on Grandiosity and on the use of marijuana, cocaine, LSD and ecstasy, while girls scored higher on tobacco use (smoking). Despite these differences, gender did not moderate the association between schemas and substance use.

Key words: Substance use, cognitive schemas, impulsivity, adolescents, stress. 


\section{INTRODUCCIÓN}

E consumo de drogas es un fenómeno relacionado con el desarrollo, que aumenta casi linealmente desde el comienzo hasta el final de la adolescencia (Young, Corley, Stalling, Rhee, Crowley y Hewitt, 2002). En el caso del alcohol o el tabaco, su inicio sería aún mayor ya que según la encuesta escolar del Plan Nacional sobre Drogas (2005), el $82 \%$ y $60 \%$ de los adolescentes consumiria estas sustancias, respectivamente. Ese inicio tan temprano en la adquisición de conductas de consumo repercutiría de manera considerable en la vida de los adolescentes (Echeburúa y Corral, 1996) y tendria múltiples consecuencias en su vida adulta (Hidalgo y Redondo, 2005).

El origen temprano del problema hace importante la identificación de los factores de riesgo implicados con el fin de establecer estrategias preventivas. El objetivo de este estudio es evaluar el papel que el estrés y algunas variables moderadoras del mismo ejercen en el consumo de drogas.

El estrés es un factor asociado a la conducta adictiva (Nadal, 2008). La adolescencia va acompañada de un aumento del estrés vital, ya que implica hacer frente a una serie de retos y nuevas obligaciones que coinciden con los cambios biológicos y físicos de la pubertad y con fluctuaciones en el funcionamiento emocional, cognitivo y social. Las presiones académicas, la imagen corporal, el desarrollo de la identidad sexual, el logro de una creciente autonomía con respecto a los padres y madres, la aceptación por parte del grupo, etc., constituyen, sin duda, fuentes potenciales de estrés en la adolescencia. Especialmente, la adolescencia media (de 14 a 16 años) se considera un periodo 'cumbre' a lo largo del ciclo vital para la ocurrencia de acontecimientos vitales estresantes (Windle y Windle, 1996). Como consecuencia, a menudo se experimenta depresión y otras emociones negativas. Esto ha llevado a sugerir que el consumo de alcohol y tabaco (Wills, 1986; Wills, McNamara, Vaccaro y Hirky, 1996) y de otras drogas (ArellanezHernández, Díaz, Wagner-Echeagaray y Pérez, 2004; Griffin, Séller, Botvin y Díaz, 2001) podrian representar intentos disfuncionales de manejo de estas dificultades de adaptación. Es por ello que se ha asociado el estrés vital como factor de riesgo para el consumo de drogas en adolescentes (Wills, 1986; Windle y Windle, 1996).

Por otro lado, desde el modelo de la Terapia Cognitiva se ha propuesto que determinados estilos cognitivos solos o en interacción con los acontecimientos estresantes actuarian como factores de riesgo para el desarrollo de problemas de conducta. En el caso de las conductas que rompen con las normas, y el consumo de drogas entra en esta categoría (Achenbach y Rescorla, 2001), algunos estudios han resaltado el papel de esquemas cognitivos relacionados tanto con la dificultad para ejercer el autocontrol como con sesgos en la visión del si mismo (Barry, Frick, Adler y Grafeman, 2007; Barry, Grafeman, Adler y Pickard, 2007; Calvete, 2008). En particular Young y Klosko (1993) propusieron la existencia de un dominio de esquemas cognitivos disfuncionales que se asociarian a problemas tales como la conducta agresiva y las adicciones. Este dominio fue denominado Límites Deteriorados e implica dificultades en el establecimiento de los límites internos y de las responsabilidades respecto a los demás. Incluye dos esquemas: Grandiosidad y Autocontrol Insuficiente. El esquema de Grandiosidad se refiere a la creencia de que uno es superior a otras personas $y$, por ello, merecedor de derechos y privilegios especiales. Se asocia con la incapacidad para aceptar los límites reales de la vida. El contenido de este esquema es similar al constructo de narcisismo disfuncional (Barry et al., 2007). Las personas con este esquema se sienten especiales e insisten en que tienen que hacer, decir o tener todo lo que quieren inmediatamente (Young, 1999). En definitiva, coincidirian con los adolescentes que Urra (2006) ha descrito como exigentes, que reclaman, demandan y piden de todo pero que no soportan un "no". Reflejaría asimismo una pobre tolerancia a la frustración, la cual ha sido propuesta por Mesa y León-Fuentes (1996) como uno de los factores de vulnerabilidad personal asociados a la conducta de consumo en adolescentes. El segundo esquema, Autocontrol Insuficiente, está muy relacionado con el anterior e implica la creencia de que uno carece de autocontrol para lograr los objetivos personales o para controlar la excesiva expresión de los impulsos propios.

McGinn y Young (1996) describieron que los esquemas cognitivos se activan por los ambientes estresantes que son relevantes a cada esquema particular. Cuando se activan, los esquemas generan emociones muy intensas en la persona y conducen directa o indirectamente a una serie de problemas psicológicos tales como depresión, sentimientos de soledad, relaciones destructivas, adicciones al alcohol y drogas, entre otros. Sin embargo, en nuestro conocimiento ningún estudio ha evaluado empíricamente el papel de los mencionados esquemas cognitivos en el consumo de drogas en general y en la adolescencia en particular. Habría que destacar, no obstante que se ha encontrado que estos esquemas desempeñan un papel importante en la ludopatía (Estévez y Calvete, 2007), y que están muy relacionados con algunos trastornos de personalidad, como por ejemplo el trastorno de personalidad narcisista, el cual si ha sido vinculado con la dependencia al alcohol (Bravo de Medina, Echeburúa y Aizpiri, 2007).

Por otro lado, los esquemas cognitivos podrian actuar como factor de riesgo para el consumo de drogas especialmente cuando se combinan con un estilo impulsivo de resolución de problemas, al igual que se ha demostrado para otras conductas antisociales (Calvete, 2008; Lochman, Powell, Whidby y Fitzgerald, 2006). Este estilo implica considerar pocas alternativas de solución de los problemas, aceptar impulsivamente la primera idea que viene a la mente y valorar de forma descuidada y poco cuidadosa las consecuencias de estas soluciones (D'Zurilla; Chang y Sanna, 2003). De esta manera, se puede proponer que los esquemas cognitivos relacionados con la falta de límites motivarían el consumo de drogas especialmente en concurrencia con dicho estilo impulsivo. Es decir, los esquemas mencionados proporcionarian contenidos cognitivos relativos al deseo de obtener gratificaciones inmediatas y el estilo impulsivo impediria la reflexión necesaria sobre las consecuencias 
de conductas de riesgo. De hecho, numerosos estudios han relacionado la impulsividad con el consumo de drogas (Fantín, 2006; Jentsch y Taylor, 1999; Lyvers, 2000; Moeller y Dougherty, 2002; Nadal, 2008). Por ejemplo, en un reciente estudio se encontró que los pacientes dependientes de alcohol presentaban un mayor nivel de impulsividad que los participantes de un grupo control clínico y un grupo control sano (Bravo de Medina et al., 2007).

Finalmente, los estudios epidemiológicos del consumo en la adolescencia han mostrado diferencias en función del sexo en el consumo de alcohol y otras sustancias (Frone, Cooper y Russell, 1994; Poulin, Hand, Boudreau y Santor, 2005). En el consumo de tabaco en la población escolar mayor de 12 años (Observatorio Vasco de Drogodependencias, 2004) se ha encontrado la existencia de un número mayor de consumidoras que de consumidores. En el resto de consumos, aunque se está encontrando una equiparación creciente, aún sigue destacando el mayor número de chicos adolescentes consumidores frente a consumidoras en alcohol (Observatorio Español sobre Drogas 2000), cannabis (Observatorio Español sobre Drogas, 2001), éxtasis y LSD (Observatorio Vasco de Drogodependencias, 2002).

También se han encontrado diferencias según el sexo en el impacto de los estresores (Hankin y Abramson, 2001) y en estilos cognitivos (Allgood-Merten, Lewinsohn, y Hops 1990; Calvete y Cardeñoso, 2005; Hankin, Abramson, Moffitt, Silva, McGee y Angell, 1998; Hankin y Abramson, 1999, 2001; Twenge y Nolan-Hoeksema, 2002; Wichstrom, 1999), mostrando los chicos puntuaciones más altas en grandiosidad y falta de autocontrol. Por este motivo, en este estudio quisimos evaluar las diferencias de género en consumo y en el modo en que el estrés y los esquemas cognitivos mencionados afectan a dicho consumo.

Enlazando con los datos mencionados, el primer objetivo de este estudio consistió en evaluar la asociación entre estrés, esquemas cognitivos, impulsividad y consumo de drogas en adolescentes. Esperamos encontrar que los esquemas cognitivos moderarían la relación entre estrés y consumo de drogas, y que los esquemas cognitivos se asociarían al consumo especialmente en combinación con un estilo impulsivo de resolución de problemas, tal y como se ha encontrado que sucede para otros problemas de conducta. Por último, el segundo objetivo consistió en analizar las diferencias según sexo en consumo de drogas y evaluar si el sexo moderaba la asociación entre esquemas cognitivos y consumo.

\section{MÉTODO}

\section{Participantes}

En este estudio participaron 657 adolescentes de Bizkaia. La edad media fue de 15.99 años (D.T. = 1.08). En cuanto al sexo, la muestra estaba compuesta por 367 chicas y 290 chicos. Los participantes provenían de 6 centros escolares, tanto públicos como privados o concertados de Bizkaia y cursaban $1^{\circ} 02^{\circ}$ de Bachillerato. Siguiendo los criterios de Arias et al. (2004), y según la información proporcionada por los centros escolares sobre ingresos económicos y nivel educativo de los padres, los niveles socioeconómicos se distribuyeron de la siguiente manera: $4 \%$ bajo, $22.5 \%$ medio-bajo, $53.5 \%$ medio y $10 \%$ medio-alto.

\section{Variables e Instrumentos de Medida}

Inventario de Consumo de Drogas en Adolescentes. Para evaluar de forma pormenorizada los hábitos de consumo en los adolescentes de la muestra se construyó un inventario específico, basado en el desarrollado por Wills, Sandy, Yaeger, Cleary y Shinar (2001). Los adolescentes tenían que indicar para una serie de sustancias (tabaco, alcohol, marihuana, cocaína, speed, LSD, éxtasis, hachis) en que medida las consumian, empleando una escala de 1 (nunca), 2 (una o dos veces en el último año), 3 (cuatro o cinco veces en el último año), 4 (unas pocas veces al mes), 5 (unas pocas veces a la semana) y 6 (diariamente).

\section{Listado de acontecimientos estresantes para} adolescentes. Para evaluar la presencia de acontecimientos vitales y estresantes y la forma en que estos son percibidos empleamos una versión adaptada a nuestra cultura del APES (Adolescent Perceived Events Scales, Escalas de Acontecimientos Percibidos para Adolescentes; Compas, Davis, Forsythe y Wagner, 1987). Mide una amplia gama de acontecimientos, incluyendo problemas académicos y familiares, así como dificultades en las relaciones amorosas y de amistad. Los adolescentes deben señalar si tales acontecimientos han sucedido en sus vidas recientemente (en los últimos seis meses). En caso afirmativo deben además evaluar en que medida fueron estresantes 0 problemáticos, empleando una escala que va desde nada estresante (0) hasta muy estresante (3). En este estudio únicamente se empleó el número de estresores sucedidos ya que la evaluación de su intensidad puede estar en parte contaminada por los estilos cognitivos (Dohrenwend, 2006).

Esquemas cognitivos. Estos fueron evaluados por el Cuestionario de Esquemas - Forma reducida (Schema Questionnaire-Short Form, SQ-SF; Young y Brown, 1994). EI SQ-SF consta de 75 ítems que deben ser contestados de acuerdo con una escala likert de seis puntos ( $1=$ totalmente falso, 2 = la mayoría de las veces falso, 3 = más verdadero que falso, 4 = en ocasiones verdadero, 5 = la mayoría de las veces verdadero, y $6=$ me describe perfectamente). En esta investigación se utilizaron las escalas correspondientes a los esquemas de Autocontrol Insuficiente y Grandiosidad. El esquema de Autocontrol Insuficiente implica un déficit en el autocontrol y en la tolerancia a la frustración para lograr los objetivos personales o para controlar la excesiva expresión de los impulsos propios (por ejemplo, "Me cuesta obligarme a terminar tareas rutinarias o aburridas"). El esquema de Grandiosidad consiste en la creencia de que se es superior a otros y, por ello, merecedor de derechos y privilegios especiales (por ejemplo, "Odio que me limiten o que no me dejen hacer lo que quiera"). 
La versión en español del SQ-SF presenta buenas propiedades psicométricas, con confirmación de su estructura factorial y con consistencia interna buena para los factores (Calvete, Estévez, López de Arroyabe y Ruíz, 2005). La versión corta utilizada en este estudio estuvo desarrollada utilizando los ítems de la versión larga del SO que han sido traducidos al español por Cid y Torrubia en colaboración con Young. El SO español ha mostrado buena fiabilidad (coeficientes alpha entre .70 y .90) y correlación con depresión, afectividad positiva y negativa, y autoestima (Cid y Torrubia, 2002).

Estilo Impulsivo/Descuidado de resolución de problemas. Se empleó el Cuestionario Revisado de Resolución de Problemas- Forma reducida (SPSI-R Short Form; D'Zurilla, Nezu y Maydeu-Olivares, 1998). La Forma Abreviada del SPSI-R es un cuestionario de autoinforme que consta de 25 items que mide las dimensiones adaptativas y disfuncionales de las dimensiones de la resolución de conflictos. Cada ítem está medido en una escala likert de 5 puntos que va desde 0 (no es cierto en absoluto para mí) a 4 (extremadamente cierto para mí). El estilo impulsivo/descuidado consiste en un patrón de resolución de problemas que se caracteriza por acciones impulsivas y descuidadas y por intentos incompletos de solucionar los problemas. Un estudio con la versión en castellano de la Forma Reducida del SPSI-R ha confirmado su estructura factorial y ha obtenido coeficientes alpha adecuados (Calvete y Cardeñoso, 2001). En este estudio, el coeficiente alpha ha sido .78.

\section{Procedimiento}

Los adolescentes completaron los cuestionarios y datos demográficos en las aulas. La aplicación del cuestionario tuvo lugar en los meses de octubre y noviembre de 2005. Las pruebas se presentaron en un cuadernillo en el que se explicaba la naturaleza del estudio. Este se presentaba como un estudio sobre las conductas y formas de pensar en la adolescencia. Se les informaba que las respuestas eran anónimas y que los datos tenían un carácter confidencial. Puesto que no se incluyen los nombres de los estudiantes en los cuestionarios, se empleó el consentimiento pasivo de padres y madres, quienes fueron advertidos y tuvieron la opción de rehusar a que su hijo participase. La aplicación duraba aproximadamente entre 40 y 50 minutos. Por otro lado, se les ofrecía a los centros escolares la posibilidad de contactar de manera telefónica o vía mail para obtener información más detallada sobre el estudio o resultados preliminares.

\section{RESULTADOS}

\section{Estresores, esquemas cognitivos, estilo impulsivo y consumo de drogas en adolescentes.}

En primer lugar, a través de regresiones múltiples jerárquicas se estudió la influencia de los esquemas cognitivos en la relación entre estresores y el consumo de drogas. En el primer paso se introdujeron los esquemas cognitivos, el número de estresores experimentados y el estilo impulsivo como variables predictoras. Siguiendo el procedimiento recomendado por Frazier, Tix y Barron (2004) se emplearon las puntuaciones $Z$ de las variables para reducir los problemas de multicolinearidad entre las variables en la ecuación de regresión. En un segundo paso se introdujeron los términos de interacción entre estresores y esquemas cognitivos, estresores y estilo impulsivo y esquemas cognitivos y estilo impulsivo.

Los resultados se muestran en la Tabla 1. Como puede observarse, tanto el número de estresores como los esquemas cognitivos de Grandiosidad y Autocontrol Insuficiente se asociaron significativamente al consumo en adolescentes. En cuanto a las interacciones los resultados muestran que la acción de los esquemas cognitivos es independiente del número de estresores experimentados y del estilo impulsivo. Sin embargo, la interacción impulsividad $x$ estresores se asocia significativamente al consumo. Para interpretar esta interacción se procedió a su representación en la Figura 1. En esta se reflejan los datos de consumo para adolescentes que puntúan bajo y alto en impulsividad y número de estresores (una desviación por debajo y una desviación por encima de la media). Tal y como puede observarse, la relación entre estresores y consumo de drogas es mayor entre los adolescentes con un estilo no impulsivo.

Tabla 1. Resumen de la regresión lineal para los esquemas cognitivos como moderadores en la relación entre estresores y consumo de drogas en adolescentes.

\begin{tabular}{|c|c|c|c|c|}
\hline Consumo drogas & B & $\mathrm{SE}$ & B & $\mathrm{t}$ \\
\hline Paso 1 & \multicolumn{4}{|c|}{$R^{2}=.13,7(4.651)=23.17^{* *}$} \\
\hline Grandiosidad & .63 & .15 & .18 & $4.13^{* *}$ \\
\hline Autocontrol Insuficiente & .26 & .16 & .10 & $2.32^{*}$ \\
\hline Impulsividad & .30 & .16 & .10 & 1.94 \\
\hline Estresores & .43 & .14 & .12 & $3.14^{*}$ \\
\hline Paso 2 & \multicolumn{4}{|c|}{$\Delta R^{2}=.14 . \Delta$ ( 9.646$)=11.4^{* *}$} \\
\hline Estresores $x$ Grandiosidad & .19 & .16 & .05 & 1.22 \\
\hline Estresores $x$ Autocontrol Insuficiente & .00 & .17 & .00 & .00 \\
\hline Estresores $x$ Impulsividad & -.43 & .16 & -.11 & $-2.73^{*}$ \\
\hline Grandiosidad $x$ Impulsividad & .12 & .15 & .04 & .81 \\
\hline Autocontrol Insuficiente $x$ Impulsividad & .05 & .15 & .01 & .31 \\
\hline
\end{tabular}

${ }^{*} p<.05 ;{ }^{* *} p<.001$

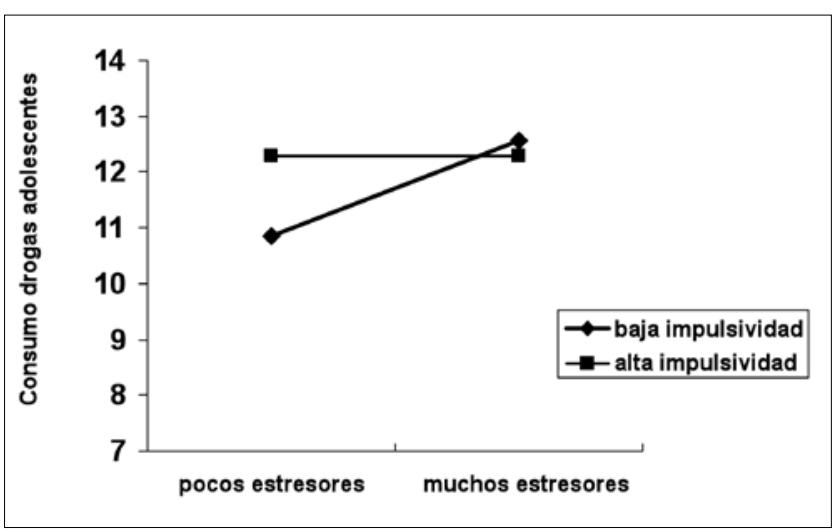

Figura. 1 Estresores, impulsividad y consumo de drogas en adolescentes. 


\section{Diferencias en consumo y esquemas cognitivos según sexo.}

La Tabla 2 muestra las puntuaciones en chicos y chicas en todas las variables del estudio, junto con los resultados de la prueba $t$ de Student. Los chicos puntuaron significativamente más alto que las chicas en el consumo en general. Más específicamente, destacaron en el consumo de marihuana, cocaina, LSD y éxtasis. Las chicas, por su parte, tuvieron puntuaciones mayores en tabaco que los chicos. No hubo diferencias según sexo en el resto de consumos. Respecto a los esquemas cognitivos e impulsividad, tan solo hubo diferencias significativas en el esquema cognitivo de Grandiosidad, siendo mayor en el caso de los chicos.

Tabla 2. Diferencias en consumo y esquemas cognitivos según género

\begin{tabular}{lccccc}
\hline & \multicolumn{3}{c}{ Hombres } & \multicolumn{2}{c}{ Mujeres } \\
& Media & DT & Media & DT & \\
\hline & 2.62 & 1.79 & 3.13 & 1.91 & $3.51^{* *}$ \\
Tabaco & 3.69 & 1.23 & 3.77 & 1.03 & 0.81 \\
Alcohol & 2.19 & 1.42 & 1.95 & 1.29 & $-2.28^{*}$ \\
Marihuana & 1.20 & 0.79 & 1.07 & 0.34 & $-2.94^{*}$ \\
Cocaina & 1.13 & 0.72 & 1.01 & 0.22 & $-3.03^{*}$ \\
LSD & 1.14 & 0.68 & 1.08 & 0.41 & -1.53 \\
Speed & 1.11 & 0.60 & 1.00 & 0.05 & $-3.49^{* *}$ \\
Éxtasis & 1.21 & 0.79 & 1.22 & 0.87 & 0.17 \\
Hachis & 0.87 & 0.73 & 0.83 & 0.55 & 0.64 \\
Drogas diseño & 12.54 & 4.23 & 11.92 & 2.69 & $-2.29^{*}$ \\
Consumo total & 7.33 & 4.12 & 6.22 & 3.40 & $-3.80^{* *}$ \\
Grandiosidad & 6.87 & 4.22 & 6.55 & 4.04 & 0.98 \\
Impulsividad & 7.97 & 3.70 & 8.25 & 3.91 & 0.96 \\
Autocontrol & & & & & \\
\hline & & & & &
\end{tabular}

${ }^{*} p<.05 ;{ }^{* *} p<.001$

\section{El sexo como moderador del impacto de los esquemas cognitivos en el consumo de drogas en la adolescencia.}

Por último, se estudió la influencia del sexo en el impacto de los esquemas cognitivos de Grandiosidad y Autocontrol Insuficiente y la Impulsividad en el consumo de drogas. Para ello se emplearon regresiones múltiples jerárquicas. En el primer paso se introdujeron el sexo (con los códigos -1 y 1 para chicas y chicos, respectivamente), y las puntuaciones $Z$ de los esquemas cognitivos y de impulsividad. En el segundo paso se introdujeron los términos de interacción.

Los resultados se muestran en la Tabla 3. Como puede observarse, ninguna de las interacciones fue estadísticamente significativa mostrando que el sexo no moderaba las relaciones entre esquemas, impulsividad y consumo.
Tabla 3.Resumen de las regresiones jerárquicas de esquemas e impulsividad moderados por el sexo en el consumo de drogas.

\begin{tabular}{|c|c|c|c|c|c|}
\hline Impulsividad & B & ET & $\beta$ & $\mathrm{T}$ & \\
\hline Paso 1 & & & & & $R^{2}=.06 . .(2.654)=21.21^{* *}$ \\
\hline Sexo & .28 & .13 & .08 & $2.11^{*}$ & \\
\hline Impulsividad & .85 & .18 & .24 & $4.64^{* *}$ & \\
\hline Paso 2 & & & & & $\Delta R^{2}=.00 . \Delta f(3.653)=14.15^{* *}$ \\
\hline Sexo $x$ impulsividad & -.08 & .27 & -.02 & 0.28 & \\
\hline Grandiosidad & $\mathrm{B}$ & ET & $\beta$ & $T$ & \\
\hline Paso 1 & & & & & $R^{2}=.082 . F(2.652)=29.31^{* *}$ \\
\hline Sexo & .16 & .13 & .05 & 1.20 & \\
\hline Grandiosidad & .81 & .20 & .28 & $4.1^{* *}$ & \\
\hline Paso 2 & & & & & $\Delta R^{2}=.002 . \mathrm{DF}(3.651)=20.1^{* *}$ \\
\hline Sexo $\times$ grandiosidad & .33 & 0.27 & .07 & 1.23 & \\
\hline $\begin{array}{l}\text { Autocontrol } \\
\text { Insuficiente }\end{array}$ & B & ET & $\beta$ & T & \\
\hline Paso 1 & & & & & $R^{2}=.00 .7(2.652)=26.64^{* *}$ \\
\hline Sexo & .34 & .13 & .10 & $2.54^{*}$ & \\
\hline $\begin{array}{l}\text { Autocontrol } \\
\text { Insuficiente }\end{array}$ & .91 & .17 & .26 & $5.20^{* *}$ & \\
\hline Paso 2 & & & & & $\Delta R^{2}=.08 . \Delta \Re(3.651)=17.74^{* *}$ \\
\hline $\begin{array}{l}\text { Sexo } \times \text { Autocontrol } \\
\text { Insuficiente }\end{array}$ & .04 & .27 & .01 & 0.15 & \\
\hline \multicolumn{6}{|c|}{${ }^{*} p<.05 ;{ }^{* *} p<.001$} \\
\hline DISCUSIÓN & & & & & \\
\hline
\end{tabular}

El primer objetivo de este estudio consistió en evaluar la asociación entre estresores, esquemas cognitivos, impulsividad y consumo de drogas en adolescentes. Los resultados, tal y como se esperaba, mostraron que el número de estresores experimentado es un factor asociado al consumo de drogas en la adolescencia. Estos resultados son consistentes con numerosos estudios que han demostrado que el estrés vital predice el consumo de drogas en adolescentes. En particular, los acontecimientos vitales negativos se han asociado al consumo de tabaco (Wills et al., 1996), alcohol (Wills, 1986; Windle y Windle, 1996) y otras drogas (Arellanez-Hernández et al., 2004; Biafora, Warheit, Vega y Gil, 1994; Unger et al., 2001). Windle y Windle (1996), además, encontraron que esta asociación puede ser diferente dependiendo de la magnitud y naturaleza de los acontecimientos estresantes.

Los esquemas cognitivos consistentes en creencias de grandiosidad y autocontrol insuficiente se asociaron significativamente al consumo de drogas. Este resultado es importante porque, aunque estudios previos han mostrado el papel del esquema de Grandiosidad y Narcisismo en varias conductas antisociales (Barry, Grafeman et al., 2007; Calvete, 2008), este es en nuestro conocimiento el primer estudio en relacionarlos con la conducta de consumo de drogas. Los mencionados esquemas reflejan en definitiva un estilo cognitivo consistente en la falta de límites para los deseos personales junto con una intolerancia a la frustración que pudiera derivarse del no cumplimiento de 
dichos deseos. Es importante resaltar que este estilo ha sido identificado por expertos, profesionales y sociedad en general como cada vez más frecuente entre adolescentes de nuestra cultura (Mesa y León-Fuentes, 1996; Urra, 2006). Es además un estilo resultante en buena medida de pautas cada vez más permisivas de crianza, las cuales fallan en el establecimiento de límites y disciplina adecuados (Young, 1999). Estos resultados tienen, por tanto, implicaciones para la intervención en las adicciones, y sugieren que las acciones formativas dirigidas a padres y madres, que resalten la importancia del establecimiento de límites y el desarrollo de la tolerancia a la frustración, pueden contribuir positivamente a la prevención primaria de conductas adictivas.

Por otro lado, en este estudio partimos de la hipótesis de que los esquemas cognitivos de límites deteriorados se asociarian al consumo de drogas especialmente en concurrencia con un estilo impulsivo de resolución de problemas. Los resultados no apoyaron esta conclusión y mostraron que ambos aspectos actuaban de forma independiente. Sin embargo, lo que si se encontró es que el estilo impulsivo moderaba la asociación entre estresores y consumo. En concreto, cuando la impulsividad es alta la relación entre estresores y consumo es menor, ya que con independencia del número de estresores estos adolescentes tienden a presentar consumo mayor, mientras que cuando el estilo es poco impulsivo son únicamente los adolescentes sometidos a muchos estresores los que presentarían consumo alto. Estos datos reflejan la importancia del estilo impulsivo de resolución de problemas y son coherentes con otros hallazgos. En el caso del alcohol, por ejemplo, el rasgo impulsividad es el que más consistentemente se ha relacionado con conductas de consumo encontrándose, además, que la impulsividad evaluada en la adolescencia predice un patrón de abuso de alcohol en la vida adulta (Cloninger, Sigvardsson, Von Knorring y Bohman, 1998; Chassin, Flora y King, 2004). Asimismo, se ha hallado que en adolescentes no consumidores de alcohol el rasgo de impulsividad predice el desarrollo posterior de consumo de alcohol (Allen, Moeller, Rhoades y Cherek, 1998).

El segundo objetivo de este estudio fue el análisis de las diferencias de sexo en el consumo y en los esquemas cognitivos. Los resultados están en la línea de otros estudios que encuentran que los adolescentes consumen más en general que las chicas adolescentes (Observatorio Español sobre Drogas, 2000, 2001; Observatorio Vasco de Drogodependencias, 2002), salvo para el consumo de tabaco, que como se ha obtenido en este estudio y en la literatura, sería más elevado para las adolescentes que para los chicos (Observatorio Vasco de Drogodependencias, 2004). Es importante señalar que entre los consumos que según este estudio son mayores en los chicos, se encuentran el consumo de éxtasis, LSD y cocaina. Los efectos de estas drogas en el caso del éxtasis (Bobes y Saiz, 2003; Morgan, 2000) y cocaína (Caballero y Alarcón, 2000) serían de euforia y en el caso del LSD serian de tipo alucinógeno (Poudevida, Farre, Roset y Camí, 2003). Si a esto se suma, que el esquema de Grandiosidad es significativamente más elevado para los chicos podríamos apuntar que el consumo en sí podría provocar el esquema cognitivo y no viceversa. Es decir, las sensaciones de euforia, grandiosidad podrían a su vez activar las estructuras mentales como el esquema cognitivo de Grandiosidad y afectar así a la percepción de los acontecimientos vitales que vive el y la adolescente.

Sin embargo, dada la naturaleza transversal de este estudio no puede evaluarse la dirección de las relaciones encontradas. Similarmente, no se puede probar la dirección de la relación entre los estresores y el consumo. Sería necesario completar este estudio con otros longitudinales (e.g., Torikka, Kaltiala-Heino et al., 2001), que permitieran demostrar que los estresores, esquemas e impulsividad predicen el consumo de drogas. Asimismo, estudios futuros deberían evaluar en que medida las variables de este estudio interactúan con otras variables identificadas en estudios previos como factores de riesgo para el consumo en adolescentes, tales como la baja autoestima y la búsqueda de sensaciones (Nadal, 2007).

A pesar de esta limitación, los resultados sugieren que los esquemas cognitivos consistentes en ideas de grandiosidad y autocontrol insuficiente se asocian al consumo de drogas. Estos resultados se unen a los estudios previos realizados sobre características de personalidad vinculadas a la conducta adictiva (e.g., Bravo de Medina et al., 2007; Fantín, 2006), mostrando como las creencias profundas desarrolladas tempranamente pueden también constituir un factor de riesgo para las adicciones. Además, los resultados indican que la ocurrencia de acontecimientos estresantes se presenta como un factor de riesgo, si bien su influencia parece estar afectada por el estilo más o menos impulsivo de afrontamiento de los mismos. Por último, los resultados muestran que el consumo de drogas sigue siendo mayor en chicos que en chicas, si bien en algunos consumos las diferencias tienden a desaparecer y en el caso del consumo de tabaco se invierten.

\section{REFERENCIAS}

Achenbach, T. M. y Rescorla, L. A. (2001). Manual for ASEBA SchoolAge Forms \& Profiles. Burlington, VT: University of Vermont. Research Center for Children, Youth, and Families.

Allen, T. J., Moeller, F. G., Rhoades, H. M. y Cherek, D. R. (1998). Impulsivity and history of drug dependence. Drug and Alcohol Dependence, 50, 137-145.

Allgood-Merten, B., Lewinsohn, P. M. y Hops, H. (1990). Sex differences and adolescent depression. Journal of Abnormal Psychology, 99, 55-63.

Arellanez-Hernández, J. L., Díaz, D. B., Wagner-Echeagaray, F. y Pérez, V. (2004). Factores psicosociales asociados con el abuso y la dependencia de drogas entre adolescentes: Análisis bivariados de un estudio de casos y controles. Salud Mental, 27, 54-64.

Arias, M.A., Sánchez, M., Gancedo, C., Martín, R., Estepa, M.R. y Díaz, M.E. (2004). Nivel socioeconómico y consumo de recursos sanitarios en atención primaria. Atención Pediátrica, 61, 292-297. 
Barry, C. T., Frick, P. J., Adler, K. K. y Grafeman, S. J. (2007). The predictive utility of narcissism among children and adolescents: Evidence for a distinction between adaptive and maladaptive narcissism. Journal of Child and Family Studies, 16, 508-521.

Barry, C. T., Grafeman, S. J., Adler, K. K. y Pickard, J. D. (2007). The relations among narcissism, self-esteem, and delinquency in a sample of at-risk adolescents. Journal of Adolescence, 30, 933942.

Biafora, F. A., Warheit, G. J., Vega, W. A. y Gil, A. G. (1994). Stressful life events and changes in substance use among a multiracial/ ethnic sample of adolescent boys. Journal of Community Psychology, 22, 296-312.

Bobes, J. y Saiz, P. A. (eds.) (2003). Monografía Drogas Recreativas. Adicciones, 15, 2.

Bravo de Medina, R., Echeburúa, E. y Aizpiri, J. (2007). Caracteristicas psicopatológicas y dimensiones de personalidad en los pacientes dependientes del alcohol: un estudio comparativo. Adicciones, 19, 372-382.

Caballero, L. y Alarcón, A. (2000). Cocaína y cocainomanía en atención primaria. En FCS (ed.). Drogas y drogodependencias en atención primaria, 2, 205-244. Madrid: Fundación Ciencias de la Salud.

Calvete, E. (2008). Justification of violence and grandiosity schemas as predictors of antisocial behavior in adolescents. Journal of Abnormal Child Psychology, 37, 108-1095.

Calvete, E. y Cardeñoso, 0. (2001). Creencias, resolución de problemas sociales y correlatos psicológicos, Psicothema, 13, 95-100.

Calvete, E. y Cardeñoso, 0. (2005). Gender differences in cognitive vulnerability to depression and behavior problems in adolescents. Journal of Abnormal Child Psychology, 33, 179-192.

Calvete, E., Estévez, A., López de Arroyabe, E. y Ruiz, P. (2005). The schema questionnaire-short form: Structure and relationship with automatic thoughts and symptoms of affective disorders. European Journal of Psychological Assessment, 21, 90-99.

Chassin, L., Flora, D. B. y King, K. M. (2004). Trajectories of alcohol and drug use and dependence from adolescence to adulthood: The Effects of parent alcoholism and personality. Journal of Abnormal Psychology, 113, 483-498.

Cid, J. y Torrubia, R. (2002, Septiembre). Schema as a construct in cognitive behavioural therapy: A study of psychometric validity using the Young Schema Questionnaire. Comunicación presentada en The XXXII European Association for Behavioural \& Cognitive Therapies. Maastricht, Netherlands.

Cloninger, C. R., Sigvardsson, S., Von Knorring, A. L. y Bohman, M. (1988). The Swedish studies of the adopted children of alcoholics: A reply to Littrell. Journal of Studies on Alcohol, 49, 500-509.

Compas, B. E., Davis, G. E., Forsythe, C. J. y Wagner, B. M. (1987). Assessment of major and daily stressful events during adolescence: The adolescent perceived events scale. Journal of Consulting and Clinical Psychology, 55, 534-541.

Dohrenwend, B. P. (2006). Inventorying stressful life events as risk factors for psychopathology: Toward resolution of the problem of intracategory variability. Psychological Bulletin, 132, 477-495.

D'Zurilla, T. J., Chang, E. C, y Sanna, L. J. (2003). Self-esteem and social problem solving as predictors of aggression in college students. Journal of Social and Clinical Psychology, 22, 424-440.

D'Zurilla, T. J., Nezu, A. M. y Maydeu-Olivares, A. (1998). Manual for the Social Problem-Solving Inventory Revised (SPSI-R), NorthTonawanda, NY: Multi-Health Systems.
Echeburúa, E. y Corral, B. (1996). Terapia de conducta en la drogadicción. En J.M. Buceta y A.M. Bueno, Tratamientos psicológicos de hábitos y enfermedades (pp. 211-243). Madrid: Pirámide.

Estévez, A. y Calvete, E. (2007). Esquemas cognitivos en personas con conducta de juego patológico y su relación con experiencias de crianza. Clínica y Salud, 18, 23- 43.

Fantín, M. B. (2006). Perfil de personalidad y consumo de drogas en adolescentes escolarizados. Adicciones, 18, 285-292.

Frazier, P. A., Tix, A. P. y Barron, K. E. (2004). Testing moderator and mediator effects in counseling psychology research. Journal of Counseling Psychology, 51, 115-134.

Frone, M. R., Cooper, M. L. y Russell, M. (1994). Stressful life events, gender, and substance use: An application of Tobit regression. Psychology of Addictive Behaviors, 8, 59-69.

Griffin, K., Séller, L., Botvin, G. J. y Díaz, T. (2001). Protective role of personal competence skills in adolescent substance use: Psychological well-being as a mediating factor. Psychology of Addictive Behaviors, 15, 194-203.

Hankin, B.L. y Abramson, L. Y. (1999). Development of gender differences in depression: Description and possible explanations. Annals of Medicine, 31, 372-379.

Hankin, B. L. y Abramson, L. Y. (2001). Development of gender differences in depression: An elaborated cognitive vulnerabilitytransactional stress theory. Psychological Bulletin, 127, 773-796.

Hankin, B. L., Abramson, L. Y., Moffitt, T. E., Silva, P. A., McGee, R. y Angell, K. E. (1998). Development of depression from preadolescence to young adulthood: Emerging gender differences in a 10-year longitudinal study. Journal of Abnormal Psychology, 107, 128-140.

Hidalgo, M. I. y Redondo, A. M. (2005). Consumo de drogas en la adolescencia. Pediatric Integral, 9, 137-155.

Jentsch, J. y Taylor, J. R. (1999). Impulsivity resulting from frontostriatal dysfunction in drug abuse: Implications for the control of behavior by reward-related stimuli. Psychopharmacology, 146, 373-390.

Lochman, J. E., Powell, N. R., Whidby, J. M., y Fitzgerald, D. P. (2006). Aggressive Children: Cognitive-Behavioral Assessment and Treatment. New York, NY: Guilford Press.

Lyvers, M. (2000). Cognition, emotion, and the alcohol-aggression relationship: Comment on Giancola. Experimental and Clinical Psychopharmacology, 8, 607-608.

McGinn, L. K. y Young, J. E. (1996). Schema-focused therapy. En P. M. Salkovskis (ed.), Frontiers of cognitive therapy (pp. 182-207). New York: Guilford Press.

Mesa, P. y León-Fuentes, J. (1996). Drogodependencias y adolescencia. Causas y consecuencias del abuso. En J. Buendia (Ed.) (1996). Psicopatología en niños y adolescentes (pp. 333-352). Madrid: Editorial Pirámide.

Moeller, F. G. y Dougherty, D. M. (2002). Impulsivity and substance abuse: What is the connection? Addictive Disorders and Their Treatment, 1, 3-10.

Morgan, M. J. (2000). Ecstasy (MDMA): A review of its possible persistent psychological effects. Psychopharmacology, 152, 230-248.

Nadal, R. (2008). La búsqueda de sensaciones y su relación con la vulnerabilidad a la adicción y al estrés. Adicciones, 20, 59-72. 
Observatorio Español sobre Drogas (2000). Encuesta Domiciliaria sobre Consumo de Drogas 1999. Madrid: Delegación del Gobierno para el Plan Nacional sobre Drogas, Ministerio del Interior.

Observatorio Español sobre Drogas (2001). Encuesta sobre drogas a población escolar, 2000. Madrid: Delegación del Gobierno para el Plan Nacional sobre Drogas, Ministerio del Interior.

Observatorio Vasco de Drogodependencias (2002). Euskadi y drogas. Vitoria: Secretaría de la Presidencia del Gobierno Vasco.

Observatorio Vasco de Drogodependencias (2004). Informe "Seis" 2003. Vitoria: Servicio Central de Publicaciones del Gobierno Vasco.

Plan Nacional sobre drogas (2005). Observatorio sobre drogas. Informe $n^{\circ}$ 5. Madrid: Delegación del Gobierno para el Plan Nacional sobre Drogas.

Poudevida S., Farré M., Roset, P. N. y Camí, J. (2003). Construcción de un cuestionario para la valoración de los efectos subjetivos de sustancias con potencial de abuso (VESSPA): evaluación del éxtasis. Adicciones, 15, 115-126.

Poulin, C., Hand D., Boudreau, B. y Santor, D. (2005). Gender differences in the association between substance use and elevated depressive symptoms in a general adolescent population. Addiction, 100, 525-535.

Torikka, A., Kaltiala-Heino, R., Rimpelä, A., Rimpelä, M. y Rantanen, P. (2001). Depression, drinking and substance use among 14- to 16-year-old Finnish adolescents. Nordic Journal of Psychiatry, 55, 351-357.

Twenge, J. M. y Nolan-Hoeksema, S. (2002). Age, gender, race, socioeconomic status, and birth cohort differences on the children's depression inventory: A meta-analysis. Journal of Abnormal Psychology, 111, 578-588.

Unger, J. B., Yan Li, C., Johnson, A., Gong, J., Chen, X., Li, C., Trinidad, D. R., Tran, N. T. y Lo, A. T. (2001) Stressful life events among adolescents in Wuhan, China: Associations with smoking, alcohol use, and depressive symptoms. International Journal of Behavioral Medicine, 8, 1-18.

Urra, J. (2006). El pequeño dictador. Cuando los padres son las víctimas. Madrid: La esfera de los libros.

Wichstrom, L. (1999). The emergence of gender difference in depressed mood during adolescence: The role of intensified gender socialization. Developmental Psychology, 35, 232-245.

Wills, T. A. (1986). Stress and coping in early adolescence: Relationships to substance use in urban school samples. Health Psychology, 5, 503-529.

Wills, T. A., McNamara, G., Vaccaro, D. y Hirky, A. E. (1996). Escalated substance use: A longitudinal grouping analysis from early to middle adolescence. Journal of Abnormal Psychology, 105, 166180.

Wills, T. A., Sandy, J. M., Yaeger, A. M., Cleary, S. D. y Shinar, O. (2001). Coping dimensions, life stress, and adolescent substance use: A latent growth analysis. Journal of Abnormal Psychology, 110, 309-323.

Windle, M. y Windle, R. C. (1996). Coping strategies, drinking motives, and stressful life events among middle adolescents. Journal of Abnormal Psychology, 105, 551-560.

Young, J. E. (2003). Young Parenting Inventory (YPI). Disponible en: www.schematherapy. com/id205.htm [2 Febrero 2008].

Young, J. E. (1999). Cognitive therapy for personality disorders: A schema-focused approach (3 ${ }^{\text {rd }}$ edition). Sarasota, FL: Professional Resources Press.

Young, J. E. y Brown, G. (1994). Young Schemas Questionnaire - S1. New York, NY: Cognitive Therapy Center.

Young, J. E. y Klosko, J. S. (1993). Reinventing your life. New York: Plume Books.

Young, S. E., Corley, R. P., Stallings, M. C., Rhee, S. H., Crowley, T. J. y Hewitt, J. K. (2002). Substance use, abuse and dependence in adolescence: Prevalence, symptom profiles and correlates. Drug and Alcohol Dependence, 68, 309-322. 